\title{
An Efficient Algorithm for Inference in Rough Set Flow Graphs
}

\author{
C.J. Butz, W. Yan, and B. Yang \\ Department of Computer Science, University of Regina, \\ Regina, Canada, S4S 0A2 \\ \{butz, yanwe111, boting\}@cs.uregina.ca
}

\begin{abstract}
Pawlak recently introduced rough set flow graphs (RSFGs) as a graphical framework for reasoning from data. No study, however, has yet investigated the complexity of the accompanying inference algorithm, nor the complexity of inference in RSFGs. In this paper, we show that the traditional RSFG inference algorithm has exponential time complexity. We then propose a new RSFG inference algorithm that exploits the factorization in a RSFG. We prove its correctness and establish its polynomial time complexity. In addition, we show that our inference algorithm never does more work than the traditional algorithm. Our discussion also reveals that, unlike traditional rough set research, RSFGs make implicit independency assumptions regarding the problem domain.
\end{abstract}

Keywords: Reasoning under uncertainty, rough set flow graphs.

\section{Introduction}

Very recently, Pawlak 7/8 introduced rough set flow graphs (RSFGs) as a graphical framework for uncertainty management. RSFGs extend traditional rough set research 910] by organizing the rules obtained from decision tables as a directed acyclic graph (DAG). Each rule is associated with three coefficients, namely, strength, certainty and coverage, which have been shown to satisfy Bayes' theorem 78. Pawlak also provided an algorithm to answer queries in a RSFG and stated that RSFGs are a new perspective on Bayesian inference [7. No study, however, has yet investigated the complexity of Pawlak's inference algorithm, nor the complexity of inference in RSFGs.

In this paper, our analysis of the traditional RSFG inference algorithm $7 / 8$. establishes that its time complexity is exponential with respect to the number of nodes in a RSFG. We then propose a new inference algorithm that exploits the factorization in a RSFG. We prove the correctness of our algorithm and establish its polynomial time complexity. In addition, we show that our algorithm never does more work than the traditional algorithm, where work is the number of additions and multiplications needed to answer a query. The analysis in this manuscript also reveals that RSFGs make implicit assumptions regarding the problem domain. More specifically, we show that the flow conservation assumption [7] is in fact a probabilistic conditional independency [13] assumption. 
It should be noted that the work here is different from our earlier work [2] in several important ways. In this manuscript, we propose a new algorithm for RSFG inference and establish its polynomial time complexity. On the contrary, we established the polynomial complexity of RSFG inference in [2] by utilizing the relationship between RSFGs and Bayesian networks [11. Another difference is that here we show that RSFG inference algorithm in $7 / 8$, has exponential time complexity, an important result not discussed in [2].

This paper is organized as follows. Section 2 reviews probability theory, RSFGs and a traditional RSFG inference algorithm [7,8]. That the traditional inference algorithm has exponential time complexity is shown in Section 3. In Section 4, we propose a new RSFG inference algorithm. We prove the correctness of this new algorithm and establish its polynomial time complexity in Section 5 . Section 6 shows that it never does more work than the traditional algorithm. In Section 7, we observe that RSFGs make independence assumptions. The conclusion is presented in Section 8 .

\section{Definitions}

In this section, we review probability theory and RSFGs.

\subsection{Probability Theory}

Let $U=\left\{v_{1}, v_{2}, \ldots, v_{m}\right\}$ be a finite set of variables. Each variable $v_{i}$ has a finite domain, denoted $\operatorname{dom}\left(v_{i}\right)$, representing the values that $v_{i}$ can take on. For a subset $X=\left\{v_{i}, \ldots, v_{j}\right\}$ of $U$, we write $\operatorname{dom}(X)$ for the Cartesian product of the domains of the individual variables in $X$, namely, $\operatorname{dom}(X)=\operatorname{dom}\left(v_{i}\right) \times$ $\ldots \times \operatorname{dom}\left(v_{j}\right)$. Each element $c \in \operatorname{dom}(X)$ is called a configuration of $X$. If $c$ is a configuration on $X$ and $Y \subseteq X$, then by $c_{Y}$ we denote the configuration on $\mathrm{Y}$ by dropping from $c$ the values of those variables not in $Y$.

A potential [12 on $\operatorname{dom}(U)$ is a function $\phi$ on $\operatorname{dom}(U)$ such that the following two conditions both hold: (i) $\phi(u) \geq 0$, for each configuration $u \in \operatorname{dom}(U)$, and (ii) $\phi(u)>0$, for at least one configuration $u \in \operatorname{dom}(U)$. For brevity, we refer to $\phi$ as a potential on $U$ rather than $\operatorname{dom}(U)$, and we call $U$, not $\operatorname{dom}(U)$, its domain [12]. By $X Y$, we denote $X \cup Y$.

A joint probability distribution (jpd) [12] on $U$ is a function $p$ on $U$ such that the following two conditions both hold: (i) $0 \leq \phi(u) \leq 1$, for each configuration $u \in U$, and (ii) $\sum_{u \in U} \phi(u)=1.0$.

Example 1. Consider five attributes Manufacturer $(M)$, Dealership $(D)$, Age $(A)$, Salary $(S)$, Position $(P)$. One jpd $p(U)$ on $U=\{M, D, A, S, P\}$ is depicted in Appendix I.

We say $X$ and $Z$ are conditionally independent [13] given $Y$, denoted $I(X, Y, Z)$, in a joint distribution $p(X, Y, Z, W)$, if

$$
p(X, Y, Z)=\frac{p(X, Y) \cdot p(Y, Z)}{p(Y)},
$$


where $p(V)$ denotes the marginal [12] distribution of a jpd $p(U)$ onto $V \subseteq U$ and $p(Y)>0$.

The following theorem provides a necessary and sufficient condition for determining when a conditional independence holds in a problem domain.

Theorem 1. [5] $I(X, Y, Z)$ iff there exist potentials $\phi_{1}$ and $\phi_{2}$ such that for each configuration $c$ on $X Y Z$ with $p\left(c_{Y}\right)>0, p(c)=\phi_{1}\left(c_{X Y}\right) \cdot \phi_{2}\left(c_{Y Z}\right)$.

Example 2. Recall the jpd $p(U)$ in Example 1 . The marginal $p(M, D, A)$ of $p(U)$ and two potentials $\phi(M, D), \phi(D, A)$ are depicted in Table 1. By definition, conditional independence $I(M, D, A)$ holds in $p(U)$ as $p(M, D, A)=\phi(M, D)$. $\phi(D, A)$.

Table 1. The marginal $p(M, D, A)$ of $p(U)$ in Example 1 and potentials $\phi(M, D)$ and $\phi(D, A)$

\begin{tabular}{|c|c|c|c|c|c|c|c|c|c|}
\hline$M$ & $D$ & $A$ & $p(M, D, A)$ & $M$ & $D$ & $\phi(M, D)$ & $D$ & $A$ & $\phi(D, A)$ \\
\hline$\overline{\text { Toyota }}$ & Alice & $\overline{\text { Old }}$ & 0.036 & $\overline{\text { Toyota }}$ & Alice & 0.120 & Alice & Old & 0.300 \\
\hline Toyota & Alice & Middle & 0.072 & Toyota & Bob & 0.060 & Alice & Middle & 0.600 \\
\hline Toyota & Alice & Young & 0.012 & Toyota & Dave & 0.020 & Alice & Young & 0.100 \\
\hline Toyota & Bob & Old & 0.024 & Honda & Bob & 0.150 & Bob & Old & 0.400 \\
\hline Toyota & Bob & Middle & 0.036 & Honda & Carol & 0.150 & Bob & Middle & 0.600 \\
\hline Toyota & Dave & Old & 0.002 & Ford & Alice & 0.050 & Carol & Middle & 0.600 \\
\hline Toyota & Dave & Middle & 0.006 & Ford & Bob & 0.150 & Carol & Young & 0.400 \\
\hline Toyota & Dave & Young & 0.012 & Ford & Carol & 0.050 & Dave & Old & 0.100 \\
\hline Honda & Bob & Old & 0.060 & Ford & Dave & 0.250 & Dave & Middle & 0.300 \\
\hline Honda & Bob & Middle & 0.090 & & & & Dave & Young & 0.600 \\
\hline Honda & Carol & Middle & 0.090 & & & & & & \\
\hline Honda & Carol & Young & 0.060 & & & & & & \\
\hline Ford & Alice & Old & 0.015 & & & & & & \\
\hline Ford & Alice & Middle & 0.030 & & & & & & \\
\hline Ford & Alice & Young & 0.005 & & & & & & \\
\hline Ford & Bob & Old & 0.060 & & & & & & \\
\hline Ford & Bob & Middle & 0.090 & & & & & & \\
\hline Ford & Carol & Middle & 0.030 & & & & & & \\
\hline Ford & Carol & Young & 0.020 & & & & & & \\
\hline Ford & Dave & Old & 0.025 & & & & & & \\
\hline Ford & Dave & Middle & 0.075 & & & & & & \\
\hline Ford & Dave & Young & 0.150 & & & & & & \\
\hline
\end{tabular}

\subsection{Rough Set Flow Graphs}

Rough set flow graphs are built from decision tables. A decision table [10] represents a potential $\phi(C, D)$, where $C$ is a set of conditioning attributes and $D$ is a decision attribute.

Example 3. Recall the five attributes $\{M, D, A, S, P\}$ from Example1. Consider the set $C=\{M\}$ of conditioning attributes and the decision attribute $D$. Then one decision table $\phi(M, D)$ is shown in Table 2. Similarly, decision tables $\phi(D, A)$, $\phi(A, S)$ and $\phi(S, P)$ are also depicted in Table 2 . 
Table 2. Decision tables $\phi(M, D), \phi(D, A), \phi(A, S)$ and $\phi(S, P)$

\begin{tabular}{ccc}
$M$ & $D$ & $\phi(M, D)$ \\
\hline Toyota & Alice & 120 \\
Toyota & Bob & 60 \\
Toyota & Dave & 20 \\
Honda & Bob & 150 \\
Honda & Carol & 150 \\
Ford & Alice & 50 \\
Ford & Bob & 150 \\
Ford & Carol & 50 \\
Ford & Dave & 250
\end{tabular}

\begin{tabular}{ccc}
$D$ & $A$ & $\phi(D, A)$ \\
\hline Alice & Old & 51 \\
Alice & Middle & 102 \\
Alice & Young & 17 \\
Bob & Old & 144 \\
Bob & Middle & 216 \\
Carol & Middle & 120 \\
Carol & Young & 80 \\
Dave & Old & 27 \\
Dave & Middle & 81 \\
Dave & Young & 162
\end{tabular}

\begin{tabular}{ccc}
$A$ & $S$ & $\phi(A, S)$ \\
\hline Old & High & 133 \\
Old & Medium & 67 \\
Old & Low & 22
\end{tabular}

Middle High 104

Middle Medium 311

Middle Low 104

Young High 26

Young Medium 52

Young Low 181

\begin{tabular}{ccc}
$S$ & $P$ & $\phi(S, P)$ \\
\hline High & Executive & 210 \\
High & Staff & 45 \\
High & Manager & 8 \\
Medium Executive & 13 \\
Medium & Staff & 387 \\
Medium & Manager & 30 \\
Low & Executive & 3 \\
Low & Staff & 12 \\
Low & Manager & 292
\end{tabular}
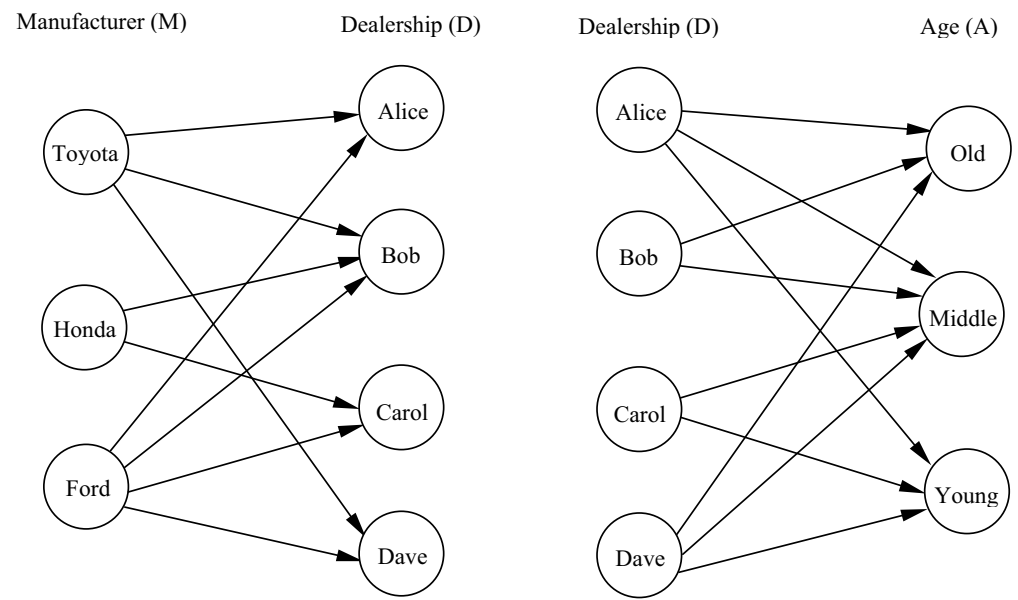

Fig. 1. The DAGs of the binary RSFGs for the decision tables $\phi(M, D)$ and $\phi(D, A)$ in Table 2 respectively. The coefficients are given in part of Table 3

Each decision table defines a binary RSFG. The set of nodes in the flow graph are $\left\{c_{1}, c_{2}, \ldots, c_{k}\right\} \cup\left\{d_{1}, d_{2}, \ldots, d_{l}\right\}$, where $c_{1}, c_{2}, \ldots, c_{k}$ and $d_{1}, d_{2}, \ldots, d_{l}$ are the values of $C$ and $D$ appearing in the decision table, respectively. For each row 
Table 3. The top two tables are the strength $\phi\left(a_{i}, a_{j}\right)$, certainty $\phi\left(a_{j} \mid a_{i}\right)$ and coverage $\phi\left(a_{i} \mid a_{j}\right)$ coefficients for the edges $\left(a_{i}, a_{j}\right)$ in Fig. 1 These two tables together with the bottom two tables are the coefficients for the edges in Fig. 2

\begin{tabular}{ccccc}
$M$ & $D$ & \multicolumn{2}{c}{$\phi_{1}(M, D) \phi_{1}(D \mid M) \phi_{1}(M \mid D)$} \\
\hline Toyota & Alice & 0.120 & 0.600 & 0.710 \\
Toyota & Bob & 0.060 & 0.300 & 0.160 \\
Toyota & Dave & 0.020 & 0.100 & 0.070 \\
Honda & Bob & 0.150 & 0.500 & 0.420 \\
Honda & Carol & 0.150 & 0.500 & 0.750 \\
Ford & Alice & 0.050 & 0.100 & 0.290 \\
Ford & Bob & 0.150 & 0.300 & 0.420 \\
Ford & Carol & 0.050 & 0.100 & 0.250 \\
Ford & Dave & 0.250 & 0.500 & 0.930
\end{tabular}

\begin{tabular}{ccccc}
$D$ & $A$ & \multicolumn{3}{c}{$\phi_{2}(D, A) \phi_{2}(A \mid D) \phi_{2}(D \mid A)$} \\
\hline Alice & Old & 0.050 & 0.300 & 0.230 \\
Alice & Middle & 0.100 & 0.600 & 0.190 \\
Alice & Young & 0.020 & 0.100 & 0.080 \\
Bob & Old & 0.140 & 0.400 & 0.630 \\
Bob & Middle & 0.220 & 0.600 & 0.420 \\
Carol & Middle & 0.120 & 0.600 & 0.230 \\
Carol & Young & 0.080 & 0.400 & 0.310 \\
Dave & Old & 0.030 & 0.100 & 0.140 \\
Dave & Middle & 0.080 & 0.300 & 0.150 \\
Dave & Young & 0.160 & 0.600 & 0.620
\end{tabular}

\begin{tabular}{ccccc}
$A$ & $S$ & $\phi_{3}(A, S)$ & $\phi_{3}(S \mid A)$ & $\phi_{3}(A \mid S)$ \\
\hline Old & High & 0.133 & 0.600 & 0.506 \\
Old & Medium & 0.067 & 0.300 & 0.156 \\
Old & Low & 0.022 & 0.100 & 0.072 \\
Middle & High & 0.104 & 0.200 & 0.395 \\
Middle Medium & 0.311 & 0.600 & 0.723 \\
Middle & Low & 0.104 & 0.200 & 0.339 \\
Young & High & 0.026 & 0.100 & 0.099 \\
Young Medium & 0.052 & 0.200 & 0.121 \\
Young & Low & 0.181 & 0.700 & 0.589
\end{tabular}

\begin{tabular}{ccccc}
$S$ & $P$ & \multicolumn{2}{c}{$\phi_{4}(S, P)$} & $\phi_{4}(P \mid S) \phi_{4}(S \mid P)$ \\
\hline High & Executive & 0.210 & 0.800 & 0.929 \\
High & Staff & 0.045 & 0.170 & 0.101 \\
High & Manager & 0.008 & 0.030 & 0.024 \\
Medium Executive & 0.013 & 0.030 & 0.058 \\
Medium & Staff & 0.387 & 0.900 & 0.872 \\
Medium & Manager & 0.030 & 0.070 & 0.091 \\
Low & Executive & 0.003 & 0.010 & 0.013 \\
Low & Staff & 0.012 & 0.040 & 0.027 \\
Low & Manager & 0.292 & 0.950 & 0.885
\end{tabular}

in the decision table, there is a directed edge $\left(c_{i}, d_{j}\right)$ in the flow graph, where $c_{i}$ is the value of $C$ and $d_{j}$ is the value of $D$. Clearly, the defined graphical structure is a directed acyclic graph (DAG). Each edge $\left(c_{i}, d_{j}\right)$ is labelled with three coefficients. The strength of $\left(c_{i}, d_{j}\right)$ is $\phi\left(c_{i}, d_{j}\right)$ obtained from the decision table. From $\phi\left(c_{i}, d_{j}\right)$, we can compute the certainty $\phi\left(d_{j} \mid c_{i}\right)$ and the coverage $\phi\left(c_{i} \mid d_{j}\right)$.

Example 4. Consider the decision tables $\phi(M, D)$ and $\phi(D, A)$ in Table 2. The DAGs of the binary RSFGs are illustrated in Fig. 1, respectively. The strength, certainty and coverage of the edges of the flow graphs in Fig. 11are shown in the top two tables of Table 3 .

In order to combine the collection of binary flow graphs into a general flow graph, Pawlak makes the flow conservation assumption [7]. This means that, for an attribute $A$ appearing as a decision attribute in one decision table $\phi_{1}\left(C_{1}, A\right)$ and also as a conditioning attribute in another decision table $\phi_{2}\left(A, D_{2}\right)$, we have

$$
\sum_{C_{1}} \phi_{1}\left(C_{1}, A\right)=\sum_{D_{2}} \phi_{2}\left(A, D_{2}\right) .
$$

Example 5. The two binary RSFGs in Example 4 satisfy the flow conservation assumption, since in Table 3, $\phi_{1}(D)=\phi_{2}(D)$. For instance, $\phi_{1}(D=$ "Alice" $)=$ $0.170=\phi_{2}(D=$ "Alice" $)$.

A rough set flow graph (RSFG) 78] is a DAG, where each edge is associated with the strength, certainty and coverage coefficients from a collection of decision tables satisfying the flow conservation assumption. 


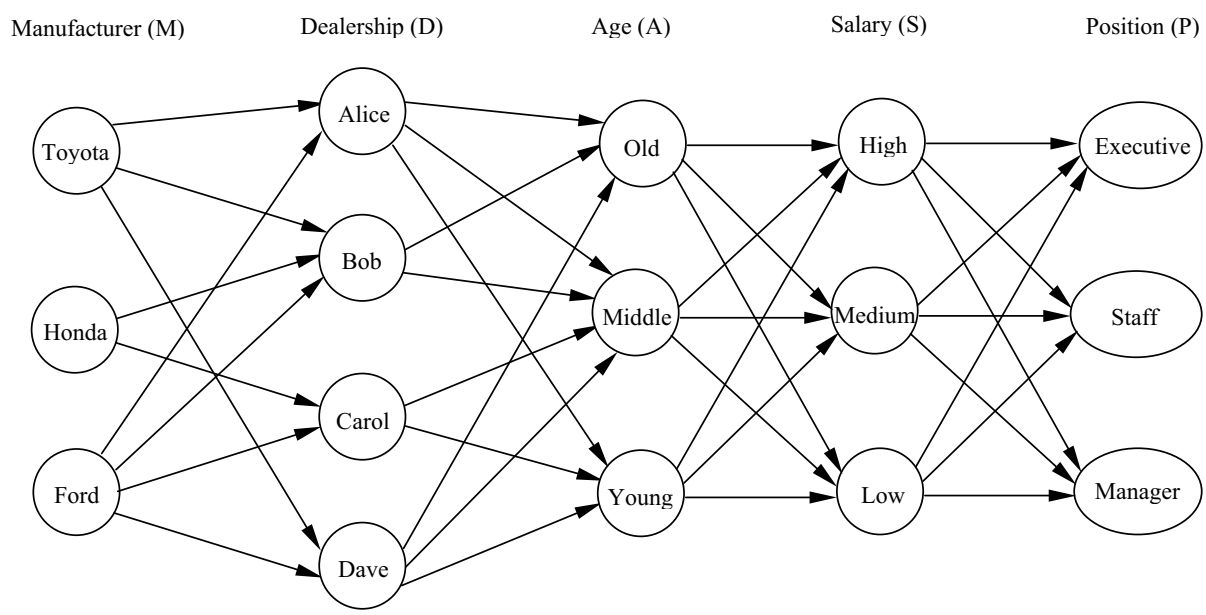

Fig. 2. The rough set flow graph (RSFG) for $\{M, D, A, S, P\}$, where the strength, certainty and coverage coefficient are given in Table 3

Example 6. The RSFG for the decision tables in Table 2 is the DAG in Fig. 2 together with the strength, certainty and coverage coefficients in Table 3 .

The task of RSFG inference is to compute a binary RSFG on $\left\{A_{i}, A_{j}\right\}$, namely, a DAG on $\left\{A_{i}, A_{j}\right\}$ and the coefficient table, denoted $\operatorname{Ans}\left(A_{i}, A_{j}\right)$, which is a table with strength, certainty and coverage columns. We use the term query to refer to any request involving strength, certainty or coverage.

Example 7. Consider a query on $\{M, P\}$ posed to the RSFG in Example 6. The answer to this query is the binary RSFG defined by Table 4 and Fig. 3 .

Pawlak proposed Algorithm 1 to answer queries in a RSFG.

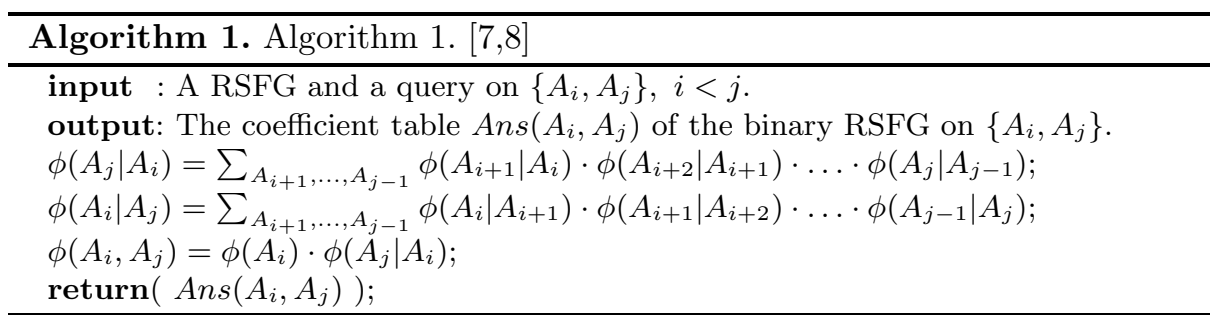

Algorithm 1 is used to compute the coefficient table of the binary RSFG on $\left\{A_{i}, A_{j}\right\}$. The DAG of this binary RSFG has an edge $\left(a_{i}, a_{j}\right)$ provided that $\phi\left(a_{i}, a_{j}\right)>0$ in $\operatorname{Ans}\left(A_{i}, A_{j}\right)$. We illustrate Algorithm 1 with Example 8 . 
Table 4. Answering a query on $\{M, P\}$ posed to the RSFG in Fig. 2 consists of this coefficient table $\operatorname{Ans}(M, P)$ and the DAG in Fig. 3

\begin{tabular}{ccrcc}
$M$ & $P$ & $\phi(M, P)$ & $\phi(P \mid M)$ & $\phi(M \mid P)$ \\
\hline Toyota & Executive & 0.053132 & 0.265660 & 0.234799 \\
Toyota & Staff & 0.095060 & 0.475300 & 0.214193 \\
Toyota & Manager & 0.051808 & 0.259040 & 0.157038 \\
Honda & Executive & 0.067380 & 0.224600 & 0.297764 \\
Honda & Staff & 0.140820 & 0.469400 & 0.317302 \\
Honda & Manager & 0.091800 & 0.306000 & 0.278259 \\
Ford & Executive & 0.105775 & 0.211550 & 0.467437 \\
Ford & Staff & 0.207925 & 0.415850 & 0.468505 \\
Ford & Manager & 0.186300 & 0.372600 & 0.564703
\end{tabular}

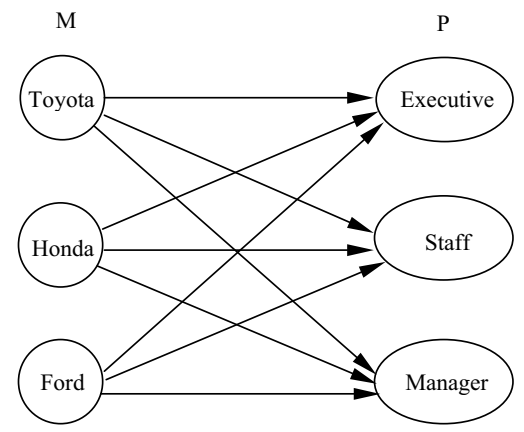

Fig. 3. Answering a query on $\{M, P\}$ posed to the RSFG in Fig. 2 consists of the coefficient table $\operatorname{Ans}(M, P)$ in Table 4 and this DAG on $\{M, P\}$

Example 8. Given a query on $\{M, P\}$ posed to the RSFG in Fig. 2. Let us focus on $M=$ "Ford" and $P=$ "Staff", which we succinctly write as "Ford" and "Staff", respectively. The certainty $\phi($ "Staff"|"Ford") is computed as:

$$
\phi(" S t a f f " \mid " F o r d ")=\sum_{D, A, S} \phi(D \mid " F o r d ") \cdot \phi(A \mid D) \cdot \phi(S \mid A) \cdot \phi(" S t a f f " \mid S) .
$$

The coverage $\phi$ ("Ford"|"Staff") is computed as:

$$
\phi(\text { "Ford" "Staff" })=\sum_{D, A, S} \phi(\text { "Ford" } \mid D) \cdot \phi(D \mid A) \cdot \phi(A \mid S) \cdot \phi(S \mid \text { "Staff" }) .
$$

The strength $\phi($ "Ford", "Staff") is computed as:

$$
\phi(\text { "Ford", "Staff" })=\phi(\text { "Ford" }) \cdot \phi(\text { "Staff"|"Ford" }) .
$$

The DAG of this binary RSFG on $\{M, P\}$ is depicted in Fig. 3 .

In Example 8, computing coefficients $\phi$ ("Ford", "Staff"), $\phi($ "Staff"|"Ford") and $\phi($ "Ford "|"Staff") in Ans $(M, P)$ in Table 4 required 181 multiplications 
and 58 additions. No study, however, has formalized the time complexity of Algorithm 1.

\section{Complexity of Traditional Algorithm in RSFG}

In this section, we establish the time complexity of Algorithm 1.

Theorem 2. Consider a RSFG on $m$ variables $U=\left\{A_{1}, A_{2}, \ldots, A_{m}\right\}$. Let $\left|\operatorname{dom}\left(A_{i}\right)\right|=n$, for $i=1, \ldots, m$. Let $\left(a_{i}, a_{i+1}\right)$ be an edge in the RSFG, where $a_{i} \in \operatorname{dom}\left(A_{i}\right), a_{i+1} \in \operatorname{dom}\left(A_{i+1}\right)$ and $i=1, \ldots, m-1$. To answer a query on $\left\{A_{i}, A_{j}\right\}$, the time complexity of Algorithm 1 is $O\left(\ln ^{l}\right)$, where $l=j-i+1$.

Proof. To compute the certainty $\phi\left(A_{j} \mid A_{i}\right)$, let

$$
\psi_{1}\left(A_{i}, A_{i+1}, \ldots, A_{j}\right)=\phi\left(A_{i+1} \mid A_{i}\right) \cdot \phi\left(A_{i+2} \mid A_{i+1}\right) \cdot \ldots \cdot \phi\left(A_{j} \mid A_{j-1}\right) .
$$

The potential $\psi_{1}\left(A_{i}, A_{i+1}, \ldots, A_{j}\right)$ has $n^{l}$ rows, since $\left|\operatorname{dom}\left(A_{i}\right)\right|=n$ for each variable. By Equation (1), computing the certainty for one row requires $l-$ 2 multiplications. Therefore, $\psi_{1}\left(A_{i}, A_{i+1}, \ldots, A_{j}\right)$ is constructed by $(l-2)\left(n^{l}\right)$ multiplications. The second step is to determine

$$
\phi\left(A_{j} \mid A_{i}\right)=\sum_{A_{i+1}, \ldots, A_{j-1}} \psi_{1}\left(A_{i}, A_{i+1}, \ldots, A_{j}\right) .
$$

There are exactly $n^{l-2}$ rows in $\psi_{1}\left(A_{i}, A_{i+1}, \ldots, A_{j}\right)$ with $A_{i}=a_{i}$ and $A_{j}=a_{j}$. Thus, computing $\phi\left(A_{j}=a_{j} \mid A_{i}=a_{i}\right)$ requires $n^{l-2}-1$ additions. Since there are $n^{2}$ configurations in $\phi\left(A_{j} \mid A_{i}\right)$, to compute $\phi\left(A_{j} \mid A_{i}\right)$ requires $\left(n^{2}\right)\left(n^{l-2}-1\right)$ additions. That is, $n^{l}-n^{2}$ additions are required for Equation (2). As shown above, the complexity to compute Equation (1) is $O\left(l n^{l}\right)$ and that to compute Equation (2) is $O\left(n^{l}\right)$. Therefore, computing the certainty $\phi\left(A_{j} \mid A_{i}\right)$ has time complexity $O\left(\ln ^{l}\right)$. It is easily seen that computing the coverage $\phi\left(A_{i} \mid A_{j}\right)$ requires exactly the same amount of work as required for computing the certainty $\phi\left(A_{j} \mid A_{i}\right)$. Thus, computing the coverage $\phi\left(A_{i} \mid A_{j}\right)$ has time complexity $O\left(\ln ^{l}\right)$. The strength $\phi\left(A_{i}, A_{j}\right)$ is defined as the product $\phi\left(A_{i}\right) \cdot \phi\left(A_{j} \mid A_{i}\right)$, which involves $n^{2}$ multiplications. Since the computation of Algorithm 1 is dominated by that for certainty (coverage), the time complexity is $O\left(\ln n^{l}\right)$.

The exponential time complexity of Algorithm 1 lies in the fact that it does not exploit the factorization during inference. However, this does not mean that Algorithm 1 is always inefficient in all practical situations.

\section{An Efficient Algorithm for RSFG Inference}

In this section, we will introduce an efficient algorithm to answer queries in a RSFG and establish its complexity.

The main idea is to exploit the factorization to eliminate variables one by one, instead of all at once as Algorithm 1 does. We focus on computing the coefficient table $\operatorname{Ans}\left(A_{i}, A_{j}\right)$ with the DAG of the output RSFG understood. 


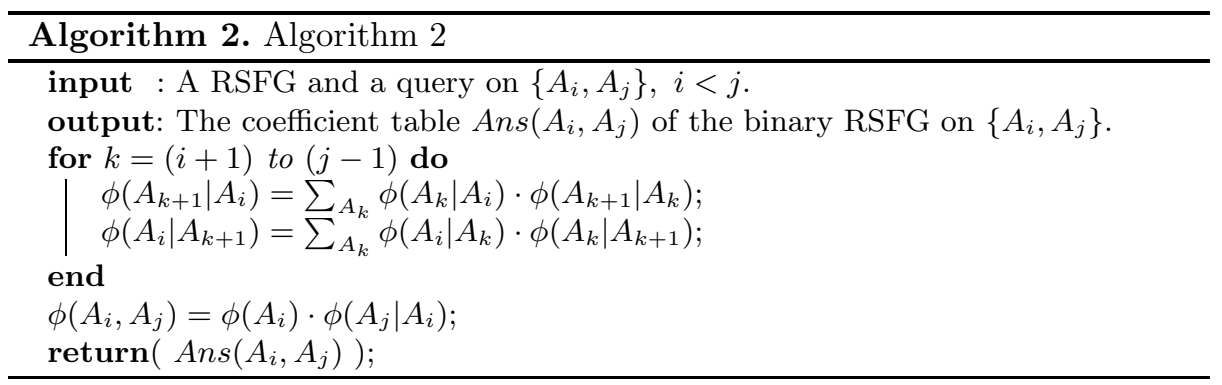

We illustrate Algorithm 2 with the following example.

Example 9. Recall Example 8. Again, we focus on the edge ("Ford" "Staff") in the DAG in Fig. 3. According to Algorithm 2, variables $\{D, A, S\}$ need be eliminated. Consider variable $D$. The certainty $\phi(A \mid$ "Ford") is

$$
\phi(A \mid \text { "Ford" })=\sum_{D} \phi(D \mid \text { "Ford" }) \cdot \phi(A \mid D),
$$

while the coverage $\phi(" F o r d " \mid A)$ is

$$
\phi(" F o r d " \mid A)=\sum_{D} \phi(" F o r d " \mid D) \cdot \phi(D \mid A) .
$$

The consequence is that variable $D$ has been eliminated, while variables $M$ and $A$ have been linked via the certainty $\phi(A \mid$ "Ford") and coverage $\phi($ "Ford" $\mid A)$. Similarly, eliminating $A$ yields $\phi(S \mid$ "Ford" $)$ and $\phi($ "Ford" $\mid S)$. Finally, consider eliminating variable $S$. The certainty $\phi(" S t a f f " \mid " F o r d ")$ is

$$
\phi(\text { "Staff" " "Ford" })=\sum_{S} \phi(S \mid \text { "Ford" }) \cdot \phi(" \text { Staff" } \mid S),
$$

while the coverage $\phi($ "Ford" " "Staff") is

$$
\phi(" \text { Ford " "Staff" })=\sum_{S} \phi(\text { "Ford" } \mid S) \cdot \phi(S \mid \text { "Staff" }) .
$$

The strength $\phi$ ("Ford", "Staff") is determined as

$$
\phi(\text { "Ford", "Staff") }=\phi(\text { "Ford" }) \cdot \phi(\text { "Staff"|"Ford"). }
$$

In Example 9, computing $\phi($ "Ford", "Staff" $), \phi($ "Staff"|"Ford") and $\phi($ "Ford "|"Staff") in Ans $(M, P)$ in Table 4 only required 45 multiplications and 30 additions. Recall that Algorithm 1 required 181 multiplications and 58 additions.

\section{Theoretical Foundation}

In this section, we show correctness of Algorithm 2 and prove Algorithm 2 is efficient by analyzing its time complexity in the worst case. 


\subsection{Correctness of the New RSFG Inference Algorithm}

Here we prove that Algorithm 2 is correct. Let us first review two well known results.

Lemma 1. [12] If $\phi$ is a potential on $U$, and $X \subseteq Y \subseteq U$, then marginalizing $\phi$ onto $Y$ and subsequently onto $X$ is the same as marginalizing $\phi$ onto $X$.

Lemma 1 indicates that a marginal can be obtained by a series of marginalizations in any order. For example,

$$
\sum_{A, B} \phi(A, B, C)=\sum_{A}\left(\sum_{B} \phi(A, B, C)\right)=\sum_{B}\left(\sum_{A} \phi(A, B, C)\right) .
$$

Lemma 2. 12] If $\phi$ is a potential on $X$ and $\psi$ is a potential on $Y$, then the marginalization of $\phi \cdot \psi$ onto $X$ is the same as $\phi$ multiplied with the marginalization of $\psi$ onto $X \cap Y$.

For instance,

$$
\sum_{C} \phi(A, B) \cdot \phi(B, C)=\phi(A, B) \cdot \sum_{C} \phi(B, C) .
$$

Now let us turn to the correctness of Algorithm 2.

Theorem 3. Given a query on $\left\{A_{i}, A_{j}\right\}$ posed to a $R S F G$ on $U=\left\{A_{1}, A_{2}, \ldots\right.$, $\left.A_{m}\right\}$, where $1 \leq i<j \leq m$. The answer produced by Algorithm 2 is correct.

Proof. We show the claim by proving that the answer table $\operatorname{Ans}\left(A_{i}, A_{j}\right)$ produced by Algorithm 2 contains the strength $\phi\left(A_{i}, A_{j}\right)$, the certainty $\phi\left(A_{j} \mid A_{i}\right)$ and the coverage $\phi\left(A_{i} \mid A_{j}\right)$ computed by Algorithm 1 . To answer the certainty $\phi\left(A_{j} \mid A_{i}\right)$, Algorithm 1 is expressed by Equation (3),

$$
\phi\left(A_{j} \mid A_{i}\right)=\sum_{A_{i+1}, A_{i+2}, \ldots, A_{j-1}} \phi\left(A_{i+1} \mid A_{i}\right) \cdot \phi\left(A_{i+2} \mid A_{i+1}\right) \cdot \ldots \cdot \phi\left(A_{j} \mid A_{j-1}\right) .
$$

By Lemma 1 and Equation (3), $\phi\left(A_{j} \mid A_{i}\right)$ is equal to

$$
\sum_{A_{i+1}} \sum_{A_{i+2}} \ldots \sum_{A_{j-2}} \sum_{A_{j-1}} \phi\left(A_{i+1} \mid A_{i}\right) \cdot \phi\left(A_{i+2} \mid A_{i+1}\right) \cdot \ldots \cdot \phi\left(A_{j} \mid A_{j-1}\right) .
$$

By Lemma 2 and Equation (4), $\phi\left(A_{j} \mid A_{i}\right)$ is equal to

$$
\sum_{A_{i+1}} \sum_{A_{i+2}} \ldots \sum_{A_{j-2}} \phi\left(A_{i+1} \mid A_{i}\right) \cdot \ldots \cdot \phi\left(A_{j-2} \mid A_{j-3}\right) \cdot \sum_{A_{j-1}} \phi\left(A_{j-1} \mid A_{j-2}\right) \cdot \phi\left(A_{j} \mid A_{j-1}\right) .
$$

By recursively using Lemma 2, Equation (5) can be rewritten as,

$$
\sum_{A_{i+1}} \phi\left(A_{i+1} \mid A_{i}\right) \cdot \sum_{A_{i+2}} \phi\left(A_{i+2} \mid A_{i+1}\right) \cdot \ldots \cdot \sum_{A_{j-1}} \phi\left(A_{j-1} \mid A_{j-2}\right) \cdot \phi\left(A_{j} \mid A_{j-1}\right) .
$$


By Equations (3) - (6), the computation of the certainty $\phi\left(A_{j} \mid A_{i}\right)$ by Algorithm 1 is expressed as,

$$
\phi\left(A_{j} \mid A_{i}\right)=\sum_{A_{i+1}} \phi\left(A_{i+1} \mid A_{i}\right) \cdot \ldots \sum_{A_{j-1}} \phi\left(A_{j-1} \mid A_{j-2}\right) \cdot \phi\left(A_{j} \mid A_{j-1}\right) .
$$

Equation (7) is the construction of the certainty $\phi\left(A_{j} \mid A_{i}\right)$ in Algorithm 2. It can be similarly shown that the strength $\phi\left(A_{i}, A_{j}\right)$ and coverage $\phi\left(A_{i} \mid A_{j}\right)$ produced by Algorithms 1 and 2 are the same.

\subsection{Complexity of the New RSFG Inference Algorithm}

In this subsection, we establish the computational complexity of Algorithm 2.

Theorem 4. Consider a RSFG on $m$ variables $U=\left\{A_{1}, A_{2}, \ldots, A_{m}\right\}$. Let $\left|\operatorname{dom}\left(A_{i}\right)\right|=n$, for $i=1, \ldots, m$. Let $\left(a_{i}, a_{i+1}\right)$ be an edge in the RSFG, where $a_{i} \in \operatorname{dom}\left(A_{i}\right), a_{i+1} \in \operatorname{dom}\left(A_{i+1}\right)$ and $i=1, \ldots, m-1$. To answer a query on $\left\{A_{i}, A_{j}\right\}$, the time complexity of Algorithm 2 is $O\left(\ln ^{3}\right)$, where $l=j-i+1$.

Proof. The certainty $\phi\left(A_{j} \mid A_{i}\right)$ is computed by eliminating each variable $A_{k}$ between $A_{i}$ and $A_{j}$ in the RSFG. For a variable $A_{k}$, Algorithm 2 first computes

$$
\psi_{2}\left(A_{k-1}, A_{k}, A_{k+1}\right)=\phi\left(A_{k} \mid A_{k-1}\right) \cdot \phi\left(A_{k+1} \mid A_{k}\right) .
$$

The potential $\psi_{2}\left(A_{k-1}, A_{k}, A_{k+1}\right)$ has $n^{3}$ rows, since $\left|d o m\left(A_{i}\right)\right|=n$ for each variable. Computing the certainty for one row requires 1 multiplication. Therefore, potential $\psi_{2}\left(A_{k-1}, A_{k}, A_{k+1}\right)$ is constructed by $n^{3}$ multiplications. The second step is to determine

$$
\phi\left(A_{k+1} \mid A_{k-1}\right)=\sum_{A_{k}} \psi_{2}\left(A_{k-1}, A_{k}, A_{k+1}\right)
$$

There are $n$ rows in $\psi_{2}\left(A_{k-1}, A_{k}, A_{k+1}\right)$ with $A_{k-1}=a_{k-1}$ and $A_{k+1}=a_{k+1}$. Thus, computing $\phi\left(A_{k+1}=a_{k+1} \mid A_{k-1}=a_{k-1}\right)$ requires $n-1$ additions. Since there are $n^{2}$ configurations in $\phi\left(A_{k+1} \mid A_{k-1}\right),\left(n^{2}\right)(n-1)$ additions are required to compute $\phi\left(A_{k+1} \mid A_{k-1}\right)$ in Equation (9). Therefore, the time complexity to compute the certainty $\phi\left(A_{k+1} \mid A_{k-1}\right)$ is $O\left(n^{3}\right)$. Since there are $l-2$ variables between $A_{i}$ and $A_{j}$, the time complexity to compute the desired certainty $\phi\left(A_{j} \mid A_{i}\right)$ has time complexity $O\left(l^{3}\right)$. Similar to the proof of Theorem 2 , it follows that the time complexity of Algorithm 2 is $O\left(l n^{3}\right)$.

Theorem 4 shows that Algorithm 2 has polynomial time complexity in the worst case. Therefore, Algorithm 2 is an efficient algorithm for RSFG inference in all practical situations. 


\section{Related Work}

In this section, we show Algorithm 2 never performs more work than Algorithm 1. To show this claim let us first characterize the computation performed by Algorithm 1 and Algorithm 2 when answering a query.

We need only focus on how the certainty $\phi\left(A_{j} \mid A_{i}\right)$ is computed from a RSFG on $U=\left\{A_{1}, A_{2}, \ldots, A_{m}\right\}$ with certainties $\phi\left(A_{2} \mid A_{1}\right), \phi\left(A_{3} \mid A_{2}\right), \ldots, \phi\left(A_{m} \mid\right.$ $\left.A_{m-1}\right)$. For simplicity, we eliminate variables in the following order: $A_{j-1}, A_{j-2}$, $\ldots, A_{i+1}$.

Algorithm 1 computes the following product $\psi_{1}\left(A_{i}, A_{i+1}, \ldots, A_{j}\right)$ :

$$
\begin{aligned}
& \psi_{1}\left(A_{i}, A_{i+1}, \ldots, A_{j}\right) \\
= & \phi\left(A_{i+1} \mid A_{i}\right) \cdot \ldots \cdot \phi\left(A_{j-2} \mid A_{j-3}\right) \cdot \phi\left(A_{j-1} \mid A_{j-2}\right) \cdot \phi\left(A_{j} \mid A_{j-1}\right)
\end{aligned}
$$

via a series of binary multiplications, namely,

$$
\begin{aligned}
& \psi_{1}\left(A_{i}, A_{i+1}, \ldots, A_{j}\right) \\
= & \phi\left(A_{i+1} \mid A_{i}\right) \cdot\left[\ldots \cdot\left[\phi\left(A_{j-2} \mid A_{j-3}\right) \cdot\left[\phi\left(A_{j-1} \mid A_{j-2}\right) \cdot \phi\left(A_{j} \mid A_{j-1}\right)\right]\right] \ldots\right] .
\end{aligned}
$$

According to Equation (10), the first multiplication is as follows,

$$
\psi_{1}\left(A_{j-2}, A_{j-1}, A_{j}\right)=\phi\left(A_{j-1} \mid A_{j-2}\right) \cdot \phi\left(A_{j} \mid A_{j-1}\right) .
$$

The intermediate multiplications are performed as follows,

$$
\psi_{1}\left(A_{k-1}, A_{k}, \ldots, A_{j}\right)=\phi\left(A_{k} \mid A_{k-1}\right) \cdot \psi_{1}\left(A_{k}, A_{k+1}, \ldots, A_{j}\right),
$$

where $k=(j-2), \ldots,(i+1)$.

After computing $\psi_{1}\left(A_{i}, A_{i+1}, \ldots, A_{j}\right)$, Algorithm 1 eliminates variables $A_{i+1}$, $A_{i+2}, \ldots, A_{j-1}$ via a series of marginalizations, namely,

$$
\sum_{A_{i+1}} \sum_{A_{i+2}} \ldots \sum_{A_{j-1}} \psi_{1}\left(A_{i}, A_{i+1}, \ldots, A_{j}\right) .
$$

An intermediate marginalization takes the form,

$$
\psi_{1}\left(A_{i}, \ldots, A_{l-1}, A_{j}\right)=\sum_{A_{l}} \psi_{1}\left(A_{i}, \ldots, A_{l-1}, A_{l}, A_{j}\right),
$$

where $l=(j-1), \ldots,(i+2)$. The final marginalization yields

$$
\phi\left(A_{j} \mid A_{i}\right)=\sum_{A_{i+1}} \psi_{1}\left(A_{i}, A_{i+1}, A_{j}\right) .
$$

Now consider how Algorithm 2 computes the certainty $\phi\left(A_{j} \mid A_{i}\right)$. As previously mentioned, Algorithm 2 eliminates variables $A_{j-1}, \ldots, A_{i+1}$ one by one. Algorithm 2 computes,

$$
\begin{aligned}
& \phi\left(A_{j} \mid A_{i}\right) \\
= & \sum_{A_{i+1}} \phi\left(A_{i+1} \mid A_{i}\right) \cdot \ldots \cdot \sum_{A_{j-2}} \phi\left(A_{j-2} \mid A_{j-3}\right) \cdot \sum_{A_{j-1}} \phi\left(A_{j-1} \mid A_{j-2}\right) \cdot \phi\left(A_{j} \mid A_{j-1}\right) .
\end{aligned}
$$


According to Equation (15), the first multiplication in Algorithm 2 is,

$$
\psi_{2}\left(A_{j-2}, A_{j-1}, A_{j}\right)=\phi\left(A_{j-1} \mid A_{j-2}\right) \cdot \phi\left(A_{j} \mid A_{j-1}\right) .
$$

Algorithm 2 then performs intermediate additions and multiplications, iteratively,

$$
\begin{aligned}
\phi\left(A_{j} \mid A_{j-2}\right) & =\sum_{A_{j-1}} \psi_{2}\left(A_{j-2}, A_{j-1}, A_{j}\right), \\
\psi_{2}\left(A_{j-3}, A_{j-2}, A_{j}\right) & =\phi\left(A_{j-2} \mid A_{j-3}\right) \cdot \phi\left(A_{j} \mid A_{j-2}\right), \\
\phi\left(A_{j} \mid A_{j-3}\right) & =\sum_{A_{j-2}} \psi_{2}\left(A_{j-3}, A_{j-2}, A_{j}\right), \\
& \vdots \\
\psi_{2}\left(A_{i}, A_{i+1}, A_{j}\right) & =\phi\left(A_{i+1} \mid A_{i}\right) \cdot \phi\left(A_{j} \mid A_{i+1}\right) .
\end{aligned}
$$

Therefore, an intermediate marginalization takes the form,

$$
\phi\left(A_{j} \mid A_{l-1}\right)=\sum_{A_{l}} \psi_{2}\left(A_{l-1}, A_{l}, A_{j}\right),
$$

where $l=(j-1), \ldots,(i+2)$. An intermediate multiplication takes the form,

$$
\psi_{2}\left(A_{k-1}, A_{k}, A_{j}\right)=\phi\left(A_{k} \mid A_{k-1}\right) \cdot \phi\left(A_{j} \mid A_{k}\right),
$$

where $k=(j-2), \ldots,(i+1)$. After these intermediate additions and multiplications, the final marginalization yields the desired certainty $\phi\left(A_{j} \mid A_{i}\right)$ :

$$
\phi\left(A_{j} \mid A_{i}\right)=\sum_{A_{i+1}} \psi_{2}\left(A_{i}, A_{i+1}, A_{j}\right)
$$

Lemma 3 shows that the intermediate potentials computed in the multiplication process of Algorithm 2 are marginalizations of the larger potentials computed in Algorithm 1. Lemma 4 shows that the intermediate potentials computed in the marginalization process of Algorithm 2 have no more rows than the marginalizations of the larger potentials computed in Algorithm 1.

Lemma 3. To answer a query on $\left\{A_{i}, A_{j}\right\}$ posed to a $R S F G$ on $U=\left\{A_{1}, A_{2}\right.$, $\left.\ldots, A_{m}\right\}, \phi\left(A_{j} \mid A_{k}\right)$ in Equation (18) of Algorithm 2 is a marginal of $\psi_{1}\left(A_{k}\right.$, $\left.A_{k+1}, \ldots, A_{j}\right)$ in Equation (12) of Algorithm 1.

Proof. By definition, the marginal of $\psi_{1}\left(A_{k}, A_{k+1}, \ldots, A_{j}\right)$ onto $\left\{A_{k}, A_{j}\right\}$ is:

$$
\sum_{A_{k+1}, \ldots, A_{j-1}} \psi_{1}\left(A_{k}, A_{k+1}, \ldots, A_{j}\right) .
$$

By Algorithm 1, Equation (20) is equal to,

$$
\sum_{A_{k+1}, \ldots, A_{j-1}} \phi\left(A_{k+1} \mid A_{k}\right) \cdot \ldots \cdot \phi\left(A_{j-1} \mid A_{j-2}\right) \cdot \phi\left(A_{j} \mid A_{j-1}\right) .
$$


By Lemmas 1] and 2, Equation (21) can be rewritten as:

$$
\sum_{A_{k+1}} \phi\left(A_{k+1} \mid A_{k}\right) \cdot \ldots \sum_{A_{j-1}} \phi\left(A_{j-1} \mid A_{j-2}\right) \cdot \phi\left(A_{j} \mid A_{j-1}\right) .
$$

By Equation (7),

$$
\phi\left(A_{j} \mid A_{k}\right)=\sum_{A_{k+1}} \phi\left(A_{k+1} \mid A_{k}\right) \cdot \ldots \sum_{A_{j-1}} \phi\left(A_{j-1} \mid A_{j-2}\right) \cdot \phi\left(A_{j} \mid A_{j-1}\right) .
$$

By Equations (20) - (23),

$$
\phi\left(A_{j} \mid A_{k}\right)=\sum_{A_{k+1}, \ldots, A_{j-1}} \psi_{1}\left(A_{k}, A_{k+1}, \ldots, A_{j}\right) .
$$

Therefore, $\phi\left(A_{j} \mid A_{k}\right)$ is the marginal of $\psi_{1}\left(A_{k}, A_{k+1}, \ldots, A_{j}\right)$ onto variables $\left\{A_{k}\right.$, $\left.A_{j}\right\}$.

Lemma 4. To answer a query on $\left\{A_{i}, A_{j}\right\}$ posed to a $R S F G$ on $U=\left\{A_{1}, A_{2}\right.$, $\left.\ldots, A_{m}\right\}, \psi_{2}\left(A_{l-1}, A_{l}, A_{j}\right)$ in Equation (17) of Algorithm 2 has no more rows than the marginal of $\psi_{1}\left(A_{i}, \ldots, A_{l-1}, A_{l}, A_{j}\right)$ in Equation (13) of Algorithm 1 onto variables $\left\{A_{l-1}, A_{l}, A_{j}\right\}$.

Proof. By definition, the marginal of $\psi_{1}\left(A_{i}, \ldots, A_{l-1}, A_{l}, A_{j}\right)$ onto variables $\left\{A_{l-1}, A_{l}, A_{j}\right\}$ is:

$$
\sum_{A_{i}, \ldots, A_{l-2}} \psi_{1}\left(A_{i}, \ldots, A_{l-1}, A_{l}, A_{j}\right) .
$$

By Algorithm 1, Equation (24) is equal to,

$$
\sum_{A_{i}, \ldots, A_{l-2}} \phi\left(A_{i+1} \mid A_{i}\right) \cdot \ldots \cdot \phi\left(A_{l-2} \mid A_{l-3}\right) \cdot \phi\left(A_{l-1} \mid A_{l-2}\right) \cdot \phi\left(A_{l} \mid A_{l-1}\right) \cdot \phi\left(A_{j} \mid A_{l}\right) .
$$

By Lemma 2, Equation (25) is equal to,

$$
\phi\left(A_{l} \mid A_{l-1}\right) \cdot \phi\left(A_{j} \mid A_{l}\right) \cdot \sum_{A_{i}, \ldots, A_{l-2}} \phi\left(A_{i+1} \mid A_{i}\right) \cdot \ldots \cdot \phi\left(A_{l-2} \mid A_{l-3}\right) \cdot \phi\left(A_{l-1} \mid A_{l-2}\right) .
$$

By Lemmas 1 and 2, Equation (26) can be rewritten as:

$$
\phi\left(A_{l} \mid A_{l-1}\right) \cdot \phi\left(A_{j} \mid A_{l}\right) \cdot \sum_{A_{i}}\left(\sum_{A_{i+1}} \phi\left(A_{i+1} \mid A_{i}\right) \cdot \ldots \cdot \sum_{A_{l-2}} \phi\left(A_{l-2} \mid A_{l-3}\right) \cdot \phi\left(A_{l-1} \mid A_{l-2}\right)\right) .
$$

By Equation (7), $\sum_{A_{i+1}} \phi\left(A_{i+1} \mid A_{i}\right) \cdot \ldots \cdot \sum_{A_{l-2}} \phi\left(A_{l-2} \mid A_{l-3}\right) \cdot \phi\left(A_{l-1} \mid A_{l-2}\right)$ yields $\phi\left(A_{l-1} \mid A_{i}\right)$. Thus, Equation (27) can be rewritten as:

$$
\phi\left(A_{l} \mid A_{l-1}\right) \cdot \phi\left(A_{j} \mid A_{l}\right) \cdot \sum_{A_{i}} \phi\left(A_{l-1} \mid A_{i}\right) .
$$


By Equation (18),

$$
\psi_{2}\left(A_{l-1}, A_{l}, A_{j}\right)=\phi\left(A_{l} \mid A_{l-1}\right) \cdot \phi\left(A_{j} \mid A_{l}\right) .
$$

Substituting Equation (29) into Equation (28), we obtain:

$$
\left.\psi_{2}\left(A_{l-1}, A_{l}, A_{j}\right) \cdot \sum_{A_{i}} \phi\left(A_{l-1} \mid A_{i}\right) .\right)
$$

By Equations (24) - (30),

$$
\sum_{A_{i}, \ldots, A_{l-2}} \psi_{1}\left(A_{i}, \ldots, A_{l-1}, A_{l}, A_{j}\right)=\psi_{2}\left(A_{l-1}, A_{l}, A_{j}\right) \cdot \sum_{A_{i}} \phi\left(A_{l-1} \mid A_{i}\right) .
$$

Therefore, $\psi_{2}\left(A_{l-1}, A_{l}, A_{j}\right)$ has no more rows than the marginal of $\psi_{1}\left(A_{i}, \ldots\right.$, $\left.A_{l-1}, A_{l}, A_{j}\right)$ onto variables $\left\{A_{l-1}, A_{l}, A_{j}\right\}$.

We use the above analysis to show the following two results. Lemma [5] says that Algorithm 2 never performs more multiplications than Algorithm 1 when answering a query. Lemma 6] says the same except for additions.

Lemma 5. Given a query on $\left\{A_{i}, A_{j}\right\}$ posed to a $R S F G$ on $U=\left\{A_{1}, A_{2}, \ldots\right.$, $\left.A_{m}\right\}$, Algorithm 2 never performs more multiplications than Algorithm 1.

Proof. It can be seen from Equations (11) and (16) that Algorithms 1 and 2 use the same number of multiplications to compute the first potential $\psi_{1}\left(A_{j-2}, A_{j-1}\right.$, $\left.A_{j}\right)$ and $\psi_{2}\left(A_{j-2}, A_{j-1}, A_{j}\right)$. Therefore, Algorithm 1 and Algorithm 2 perform the same number of multiplications provided that precisely two potentials need be multiplied to answer a query. On the other hand, Algorithm 2 never performs more multiplications than Algorithm 1 provided that there are at least three potentials to be multiplied. By Lemma [3, $\phi\left(A_{j} \mid A_{k}\right)$ is the marginal of $\psi_{1}\left(A_{k}, A_{k+1}, \ldots, A_{j}\right)$ onto $\left\{A_{k}, A_{j}\right\}$. Therefore, all multiplications in Equation (18) performed by Algorithm 2 for computing the certainty $\phi\left(A_{j} \mid A_{i}\right)$ must necessarily be performed in Equation (12) by Algorithm 1. It can be similarly shown that Algorithm 2 never performs more multiplications than Algorithm 1 when computing the strength $\phi\left(A_{i}, A_{j}\right)$ or coverage $\phi\left(A_{i} \mid A_{j}\right)$. Therefore, Algorithm 2 never performs more multiplications than Algorithm 1 when answering a query.

Lemma 6. Given a query on $\left\{A_{i}, A_{j}\right\}$ posed to a $R S F G$ on $U=\left\{A_{1}, A_{2}, \ldots\right.$, $\left.A_{m}\right\}$, Algorithm 2 never performs more additions than Algorithm 1.

Proof. It can be seen from Equations (14) and (19) that Algorithms 1 and 2 use the same number of additions to eliminate the last variable $A_{i+1}$ from the potential $\psi_{1}\left(A_{i}, A_{i+1}, A_{j}\right)$ and $\psi_{2}\left(A_{i}, A_{i+1}, A_{j}\right)$. Therefore, Algorithm 1 and Algorithm 2 perform the same number of additions provided that precisely one variable need be eliminated to answer a query. On the other hand, Algorithm 2 never performs more additions than Algorithm 1, provided that there are at 
least two variables to be eliminated. By Lemma 4, $\psi_{2}\left(A_{l-1}, A_{l}, A_{j}\right)$ has no more rows than the marginal of $\psi_{1}\left(A_{i}, \ldots, A_{l-1}, A_{l}, A_{j}\right)$ onto $\left\{A_{l-1}, A_{l}, A_{j}\right\}$. Therefore, summing out $A_{l}$ from $\psi_{2}\left(A_{l-1}, A_{l}, A_{j}\right)$ combines no more rows than needed from $\psi_{1}\left(A_{i}, \ldots, A_{l-1}, A_{l}, A_{j}\right)$. Since combining $n$ rows requires $n-1$ additions, Algorithm 2 never performs more additions than Algorithm 1 for computing the certainty $\phi\left(A_{j} \mid A_{i}\right)$. That Algorithm 2 never performs more additions than Algorithm 1 when computing the strength $\phi\left(A_{i}, A_{j}\right)$ or coverage $\phi\left(A_{i} \mid A_{j}\right)$ follows in a similar fashion. Therefore, Algorithm 2 never performs more additions than Algorithm 1 when answering a query.

Lemmas [5] and 6] indicate that Algorithm 2 never performs more work than Algorithm 1.

\section{Other Remarks on Rough Set Flow Graphs}

One salient feature of rough sets is that they serve as a tool for uncertainty management without making assumptions regarding the problem domain. On the contrary, we establish in this section that RSFGs, in fact, make implicit independency assumptions regarding the problem domain.

Two tables $\phi_{1}\left(A_{i}, A_{j}\right)$ and $\phi_{2}\left(A_{j}, A_{k}\right)$ are pairwise consistent [3]13], if

$$
\phi_{1}\left(A_{j}\right)=\phi_{2}\left(A_{j}\right)
$$

Example 10. In Table [3, $\phi_{1}(M, D)$ and $\phi_{2}(D, A)$ are pairwise consistent. For instance, $\phi_{1}(D=$ "Alice" $)=0.170=\phi_{2}(D=$ "Alice" $)$.

Consider $m-1$ potentials $\phi_{1}\left(A_{1}, A_{2}\right), \phi_{2}\left(A_{2}, A_{3}\right), \ldots, \phi_{m-1}\left(A_{m-1}, A_{m}\right)$, such that each consecutive pair is pairwise consistent, namely,

$$
\phi_{i}\left(A_{i+1}\right)=\phi_{i+1}\left(A_{i+1}\right),
$$

for $i=1,2, \ldots, m-2$. Observe that the schemas of these decision tables form an acyclic hypergraph [1]. Dawid and Lauritzen [3] have shown that if a given set of potentials satisfies Equation (32) and are defined over an acyclic hypergraph, then the potentials are marginals of a unique potential $\phi\left(A_{1}, A_{2}, \ldots, A_{m}\right)$, defined as:

$$
\phi\left(A_{1}, A_{2}, \ldots, A_{m}\right)=\frac{\phi_{1}\left(A_{1}, A_{2}\right) \cdot \phi_{2}\left(A_{2}, A_{3}\right) \cdot \ldots \cdot \phi_{m-1}\left(A_{m-1}, A_{m}\right)}{\phi_{1}\left(A_{2}\right) \cdot \ldots \cdot \phi_{m-2}\left(A_{m-1}\right)} .
$$

In 7/8, the flow conservation assumption is made. This means that a given set of $m-1$ decision tables $\phi_{1}\left(A_{1}, A_{2}\right), \phi_{2}\left(A_{2}, A_{3}\right), \ldots, \phi_{m-1}\left(A_{m-1}, A_{m}\right)$ satisfies Equation (32). By [3], these potentials are marginals of a unique potential $\phi\left(A_{1}, A_{2}, \ldots, A_{m}\right)$ defined by Equation (33), which we will call the collective potential. The collective potential $\phi\left(A_{1}, A_{2}, \ldots, A_{m}\right)$ represents the problem domain from a rough set perspective.

In order to test whether independencies are assumed to hold, it is necessary to normalize $\phi\left(A_{1}, A_{2}, \ldots, A_{m}\right)$. (Note that the normalization process has been 
used in [78.) Normalizing $\phi\left(A_{1}, A_{2}, \ldots, A_{m}\right)$ yields a jpd $p\left(A_{1}, A_{2}, \ldots, A_{m}\right)$ by multiplying $1 / N$, where $\mathrm{N}$ denotes the number of all cases. It follows from Equation (33) that

$$
\begin{aligned}
p\left(A_{1}, A_{2}, \ldots, A_{m}\right) & =\frac{1}{N} \cdot \phi\left(A_{1}, A_{2}, \ldots, A_{m}\right) \\
& =\frac{1}{N} \cdot \frac{\phi_{1}\left(A_{1}, A_{2}\right) \cdot \phi_{2}\left(A_{2}, A_{3}\right) \cdot \ldots \cdot \phi_{m-1}\left(A_{m-1}, A_{m}\right)}{\phi_{1}\left(A_{2}\right) \cdot \ldots \cdot \phi_{m-2}\left(A_{m-1}\right)} .
\end{aligned}
$$

We now show that RSFGs make implicit independency assumptions regarding the problem domain.

Theorem 5. Consider a RSFG defined by $m-1$ decision tables $\phi_{1}\left(A_{1}, A_{2}\right)$, $\phi_{2}\left(A_{2}, A_{3}\right), \ldots, \phi_{m-1}\left(A_{m-1}, A_{m}\right)$. Then $m-2$ probabilistic independencies $I\left(A_{1}, A_{2}, A_{3} \ldots A_{m}\right), I\left(A_{1} A_{2}, A_{3}, A_{4} \ldots A_{m}\right), \ldots, I\left(A_{1} \ldots A_{m-2}, A_{m-1}, A_{m}\right)$ are satisfied by the jpd $p\left(A_{1}, A_{2}, \ldots, A_{m}\right)$, where $p\left(A_{1}, A_{2}, \ldots, A_{m}\right)$ is the normalization of collective potential $\phi\left(A_{1}, A_{2}, \ldots, A_{m}\right)$ representing the problem domain.

Proof. Consider $I\left(A_{1}, A_{2}, A_{3} \ldots A_{m}\right)$. By Equation (34), let

$$
\phi^{\prime}\left(A_{1}, A_{2}\right)=\phi_{1}\left(A_{1}, A_{2}\right)
$$

and

$$
\phi^{\prime \prime}\left(A_{2}, A_{3}, \ldots, A_{m}\right)=\frac{1}{N} \cdot \frac{\phi_{2}\left(A_{2}, A_{3}\right) \cdot \ldots \cdot \phi_{m-1}\left(A_{m-1}, A_{m}\right)}{\phi_{1}\left(A_{2}\right) \cdot \ldots \cdot \phi_{m-2}\left(A_{m-1}\right)} .
$$

By substituting Equations (35) and (36) into Equation (34),

$$
p\left(A_{1}, A_{2}, \ldots, A_{m}\right)=\phi^{\prime}\left(A_{1}, A_{2}\right) \cdot \phi^{\prime \prime}\left(A_{2}, A_{3}, \ldots, A_{m}\right) .
$$

By Theorem 11. Equation (37) indicates that $I\left(A_{1}, A_{2}, A_{3} \ldots A_{m}\right)$ holds. It can be similarly shown that $I\left(A_{1} A_{2}, A_{3}, A_{4} \ldots A_{m}\right), \ldots, I\left(A_{1} \ldots A_{m-2}, A_{m-1}, A_{m}\right)$ are also satisfied by the jpd $p\left(A_{1}, A_{2}, \ldots, A_{m}\right)$.

Example 11. Decision tables $\phi(M, D), \phi(D, A), \phi(A, S)$ and $\phi(S, P)$ in Table 2 satisfy Equation (32) and are defined over an acyclic hypergraph $\{M D, D A, A S$, $S P\}$. This means they are marginals of a unique collective potential,

$$
\phi(M, D, A, S, P)=\frac{\phi(M, D) \cdot \phi(D, A) \cdot \phi(A, S) \cdot \phi(S, P)}{\phi(D) \cdot \phi(A) \cdot \phi(S)} .
$$

The normalization of $\phi(M, D, A, S, P)$ is a jpd $p(M, D, A, S, P)$,

$$
p(M, D, A, S, P)=\frac{1}{1000} \cdot \frac{\phi(M, D) \cdot \phi(D, A) \cdot \phi(A, S) \cdot \phi(S, P)}{\phi(D) \cdot \phi(A) \cdot \phi(S)},
$$

where the number of all cases $N=1000$. To show $I(M, D, A S P)$ holds, let

$$
\phi^{\prime}(M, D)=\phi(M, D)
$$


and

$$
\phi^{\prime \prime}(D, A, S, P)=\frac{1}{1000} \cdot \frac{\phi(D, A) \cdot \phi(A, S) \cdot \phi(S, P)}{\phi(D) \cdot \phi(A) \cdot \phi(S)} .
$$

Substituting Equations (40) and (41) into Equation (39),

$$
p(M, D, A, S, P)=\phi^{\prime}(M, D) \cdot \phi^{\prime \prime}(D, A, S, P) .
$$

By Theorem 1, the independence $I(M, D, A S P)$ holds in $p(M, D, A, S, P)$. It can be similarly shown that $I(M D, A, S P)$ and $I(M D A, S, P)$ are also satisfied by $p(M, D, A, S, P)$.

The important point is that the flow conservation assumption [7] used in the construction of RSFGs implicitly implies probabilistic conditional independencies holding in the problem domain.

\section{Conclusion}

Pawlak 78, recently introduced the notion of rough set flow graph (RSFGs) as a graphical framework for reasoning from data. In this paper, we established that the RSFG inference algorithm suggested in [7/8] has exponential time complexity. The root cause of the computational explosion is a failure to exploit the factorization defined by a RSFG during inference. We proposed a new RSFG algorithm exploiting the factorization. We showed its correctness and established its time complexity is polynomial with respect to number of nodes in a RSFG. In addition, we showed that it never performs more work than the traditional algorithm [78]. These are important results, since they indicate that RSFGs are an efficient framework for uncertainty management. Finally, our study has revealed that RSFGs, unlike previous rough set research, make implicit independency assumptions regarding the problem domain. Future work will report on the complexity of the inference in generalized RSFGs [4]. As the order in which variables are eliminated affects the amount of computation performed [6], we will also investigate this issue in RSFGs.

\section{Acknowledgments}

The authors would like to thank A. Skowron for constructive comments and suggestions.

\section{References}

1. Beeri, C., Fagin, R., Maier, D. and Yannakakis, M.: On The Desirability of Acyclic Database Schemes. Journal of the ACM, 30(3) (1983) 479-513

2. Butz, C.J., Yan, W. and Yang, B.: The Computational Complexity of Inference Using Rough Set Flow Graphs. The Tenth International Conference on Rough Sets, Fuzzy Sets, Data Mining, and Granular Computing, Vol. 1 (2005) 335-344 
3. Dawid, A.P. and Lauritzen, S.L.: Hyper Markov Laws in The Statistical Analysis of Decomposable Graphical Models. The Annals of Satistics, Vol. 21 (1993) 1272-1317

4. Greco, S., Pawlak, Z. and Slowinski, R.: Generalized Decision Algorithms, Rough Inference Rules and Flow Graphs. The Third International Conference on Rough Sets, and Current Trends in Computing (2002) 93-104

5. Hajek, P., Havranek T. and Jirousek R.: Uncertain Information Processing in Expert System. (1992)

6. Madson, A.L. and Jensen, F.V.: Lazy Propagation: A Junction Tree Inference Algorithm based on Lazy Evaluation, Artificial Intelligence, 113 (1-2) (1999) 203245.

7. Pawlak, Z.: Flow Graphs and Decision Algorithms. The Ninth International Conference on Rough Sets, Fuzzy Sets, Data Mining, and Granular Computing (2003) $1-10$

8. Pawlak, Z.: In Pursuit of Patterns in Data Reasoning from Data - The Rough Set Way. The Third International Conference on Rough Sets, and Current Trends in Computing (2002) 1-9

9. Pawlak, Z.: Rough Sets. International Journal of Computer and Information Sciences, Vol. 11, Issue 5 (1982) 341-356

10. Pawlak, Z.: Rough Sets: Theoretical Aspects of Reasoning about Data. Kluwer Academic (1991)

11. Pearl, J.: Probabilistic Reasoning in Intelligent Systems: Networks of Plausible Inference. Morgan Kaufmann, San Francisco, California (1988)

12. Shafer, G.: Probabilistic Expert Systems. Society for the Institute and Applied Mathematics, Philadelphia (1996)

13. Wong, S.K.M., Butz, C.J. and Wu, D.: On the Implication Problem for Probabilistic Conditional Independency, IEEE Transactions on Systems, Man, and Cybernetics, Part A: Systems and Humans, Vol. 30, Issue 6. (2000) 785-805 


\section{Appendix I}

Table 5. A jpd $p(U)$ is shown in Tables [5 and 6 where $U=\{M, D, A, S, P\}$

\begin{tabular}{|c|c|c|c|c|c|c|c|c|c|c|}
\hline$M$ & $A$ & $S$ & $P$ & $p(U)$ & $M$ & $D$ & $A$ & $S$ & $P$ & $p(U)$ \\
\hline oyota Alice & Old & High & Executive & 0.017280 & Toyota & Dave & Old & Tedium & Staff & .00054 \\
\hline & Old & & & & & & & & & \\
\hline & Old & & & & & & & & Executive & \\
\hline & Old & Medium & Execu & 0.000324 & & & Old & & & \\
\hline Alice & Old & Medium & & 0.009720 & & & Old & & & \\
\hline Alice & Old & & Man & & & & Middle & & Exec & \\
\hline Alice & Old & & Exec & & & & & & & \\
\hline Alice & Old & & & & & & & High & & \\
\hline Alice & Old & & $\mathrm{Ma}$ & & & & & Medium & Exec & \\
\hline & Middle & High & Exe & & & & & & & \\
\hline & Middle & High & & & & & & & $\mathrm{Ma}$ & \\
\hline & & High & & & & & & & Exec & \\
\hline & & & $\mathrm{E}$ & & & & & & & \\
\hline & & & & & & & & & & \\
\hline & & & & & & & & & Exe & \\
\hline & & & Exec & & & & & & & \\
\hline & & & & & & & & & & \\
\hline & & & & & & & & & & \\
\hline & & & & & & & & & & \\
\hline & & & & & & & & & tora & \\
\hline & & & & & & & & & & \\
\hline & & & & & & & & & & \\
\hline & & & & & & & & & & \\
\hline & & & & & & & & & & \\
\hline & & & & & & & & & & \\
\hline & & & & & & & & & & \\
\hline & & & & & & & & & & \\
\hline & & & & & & & & & & \\
\hline & & & & & & & & & & \\
\hline b & & & & & & & & & & \\
\hline & & & & & & & & & & \\
\hline & & & & & & & & & & 70 \\
\hline & & & & & & & & & & \\
\hline $\mathrm{B}$ & & & & & & & & & & 6 \\
\hline $\mathrm{B}$ & & & & & & & & & & 54 \\
\hline $\mathrm{B}$ & & & & & & & & & & 52 \\
\hline & & & & & & & & & & 50 \\
\hline $\mathrm{B}$ & & & & & & & & & & \\
\hline Bob & & & & & & & & Low & & 18 \\
\hline & & & & & & & & & & 72 \\
\hline & & & & & & & & & & 710 \\
\hline & & & & & & & & High & Exe & 140 \\
\hline $\mathrm{Bo}$ & & & Exe & & & & & & & 306 \\
\hline a Bob 1 & & & & & & & & High & ager & 0.00054 \\
\hline a Bob I & & & & & & & & & & \\
\hline Dave & & & & & & & & & & 0.04860 \\
\hline Dave & & & & & & & & Medium & Manager & 0.00378 \\
\hline Dave & Old & High & & 0.000036 & & & & Low & & \\
\hline a Dave & & & & & & & & & Staff & \\
\hline
\end{tabular}


Table 6. A jpd $p(U)$ is shown in Tables 5 and 6 where $U=\{M, D, A, S, P\}$

\begin{tabular}{|c|c|c|c|c|c|c|c|c|c|c|}
\hline$M$ & $D$ & $A$ & $S$ & $P$ & $p(U)$ & $M$ & $A$ & $S$ & $P$ & $p(U)$ \\
\hline Honda & & Middle & Low & Tanager & 0.017100 & Ford & iddle & ediu & Staff & \\
\hline Hond & Carc & Young & High & Execu & 0.004800 & Ford Bob & Midd & Mediu & Manager & 0.003780 \\
\hline Hond & Carc & Young & High & Staff & 0.001020 & Ford Bob & Middle & Low & Executi & 0.000180 \\
\hline Hor & Carc & Young & High & Manager & 0.000180 & Ford Bob & Middle & Low & Staff & 0.000720 \\
\hline Hо & Car & & Mediur & Executi & 0.000360 & Ford Bob & Middle & Low & Manager & .017100 \\
\hline & Car & & Iediu & Staff & 0.010800 & Ford Caro & Middle & High & Executi & 800 \\
\hline & Car & & Mediu & Manager & 840 & Ford Car & Ciddle & High & Staff & 01020 \\
\hline & $\mathrm{Ca}$ & Young & Low & Execu & 420 & Ford Car & Middle & High & Manager & \\
\hline & & Young & Low & Staff & 1680 & $\mathrm{Ce}$ & 100 & Mediur & Executi & \\
\hline & & Young & Low & Manager & 39900 & Ford & 11da & Iediu & Staff & \\
\hline Ford & Alice & Old & High & Exec & 7200 & Fo & Ciddle & Mediu & Manage & 00 \\
\hline Ford & Alice & Old & High & Staff & 0.001530 & Forc & Giddle & Low & Executi & \\
\hline Ford & Alice & Old & High & Mana & 0.000270 & Ford Car & Middle & Low & Staff & 240 \\
\hline Ford & Alice & Old & Mediur & Execr & 0.000135 & Ford & Middle & Low & Manager & 700 \\
\hline Ford & Alice & Old & Medium & Staff & 0.004050 & Fol & Young & High & Executiv & 600 \\
\hline Ford & Alice & Old & Medium & Mana & 0.000315 & For & Young & High & Staff & 0.000340 \\
\hline Ford & Alice & Old & Low & Exec & 0.000015 & For & Young & High & Manage & 0.000060 \\
\hline Ford & Alice & Old & Low & Staff & 0.000060 & Ford & Young & Medius & Executiv & 120 \\
\hline Ford & Alice & Old & Low & Mana & 1425 & $\mathrm{Fol}$ & Young & Mediu & Staff & 0.003600 \\
\hline Ford & Alice & Middle & High & Execut & 0.004800 & For & Young & Medium & Manager & 0.000280 \\
\hline Ford & Alice & Middle & High & Staff & 0.001020 & Ford Caro & Young & Low & Executiv & 0.000140 \\
\hline Ford & Alice & Middle & High & Manager & 0.000180 & Ford Caro & Young & Low & Staff & 0.000560 \\
\hline Ford & Alice & Middle & Medium & Executiv & 0.000540 & Ford Carol & Young & Low & Manager & 0.013300 \\
\hline Ford & Alice & Middle & Medium & Staff & 0.016200 & Ford Dave & Old & High & Executiv & 0.012000 \\
\hline Ford & Alice & Middle & Medium & Manager & 0.00 & Ford Dave & Old & High & Staff & 2550 \\
\hline Ford & Alice & Middle & Low & Executiv & 0.00 & Ford Dave & Old & High & Manager & 0.000450 \\
\hline Ford & Alice & Middle & Low & Staff & 0240 & Ford Dave & Old & Medium & Executiv & 0.000225 \\
\hline Ford & Alice & Middle & Low & Manager & 0.00 & Ford Dave & Old & Medium & Staff & 0.006750 \\
\hline Ford & Alice & Young & High & Executiv & 0.00 & Ford Dave & Old & Medium & Manager & 0.000525 \\
\hline Ford & Alice & Young & High & Staff & 0.000085 & Ford Dave & Old & Low & Executive & 0.000025 \\
\hline Ford & Alice & Young & High & Manager & 0.000015 & Ford Dave & Old & Low & Staff & 0.000100 \\
\hline Ford & Alice & Young & Medium & Executive & 0.0 & Ford Dave & Old & Low & Manager & 0.002375 \\
\hline Ford & Alice & Young & Medium & Staff & 0.000900 & Ford Dave & Middle & High & Executive & 2000 \\
\hline Ford & Alice & Young & Medium & Manager & 0.000070 & Ford Dave & Middle & High & Staff & 02550 \\
\hline Ford & Alice & Young & Low & Executiv & 0.0 & Ford Dave & Middle & High & Manager & 0.000450 \\
\hline Ford & Alice & Young & Low & Staff & 0.0 & Ford Dave & Middle & Medium & Executive & 1350 \\
\hline Ford & Alice & Young & Low & Manager & 0.00 & Ford Dave & Middle & Medium & Staff & 0.040500 \\
\hline Ford & Bob & Old & High & Executiv & 0.0 & Ford Dave & Middle & Medium & Manager & 0.003150 \\
\hline Ford & Bob & Old & High & Staff & 0.00 & Ford Dave & Middle & Low & Executive & 0.000150 \\
\hline Ford & Bob & Old & High & Manager & 0.00 & Ford Dave & Middle & Low & Staff & 0600 \\
\hline Ford & Bob & Old & Medium & Executive & 0.000540 & Ford Dave & Middle & Low & Manager & 0.014250 \\
\hline Ford & Bob & Old & Medium & Staff & 0.016200 & Ford Dave & Young & High & Executive & 0.012000 \\
\hline Ford & Bob & Old & Medium & Manager & 0.001260 & Ford Dave & Young & High & Staff & 0.002550 \\
\hline Ford & Bob & Old & Low & Executiv & 0.000060 & Ford Dave & Young & High & Manager & 0.000450 \\
\hline Ford & Bob & Old & Low & Staff & 0.000240 & Ford Dave & Young & Medium & Executive & 0.000900 \\
\hline Ford & Bob & Old & Low & Manager & 0.005700 & Ford Dave & Young & Medium & Staff & 0.027000 \\
\hline Ford & Bob & Middle & High & Exec & 0.014400 & Ford Dave & Young & Medium & Manager & 0.002100 \\
\hline Ford & Bob & Middle & High & Staff & 0.003060 & Ford Dave & Young & Low & Executive & 0.001050 \\
\hline & Bob & Middle & High & Manager & 0.000540 & Ford Dave & Young & Low & Staff & 0.004200 \\
\hline Ford & Bob & Middle & Mediun & Executive & 0.001620 & Ford Dave & Young & Low & Manager & 0.099750 \\
\hline
\end{tabular}

